\title{
On the Retention Function for Autobiographical Memory
}

\author{
David C. Rubin
}

\author{
Duke University
}

\begin{abstract}
College undergraduates were asked to record events from their lives, and then to date those events. Data were collected from groups of subjects using a set of cue words to prompt the events, from individual subjects, for individual cue words, from groups of subjects using no cue words, and from subjects who kept diaries. If it is assumed that the subjects encoded an equal number of events from each day of their lives, the distribution of events recorded as a function of time can be viewed as a retention function. The data from all experiments provided an excellent fit to the single-trace fragility function proposed by Wickelgren to account for more traditional laboratory learning experiments. Taken together these experiments indicate that the retention function is not an artifact of summing different functions produced by individual subjects or cue words and that the episodes recorded are, for the most part, accurately dated memories of actual events. Thus, episodic memory of a naturalistic, autobiographical nature and episodic memory for lists appear to have the same retention properties.
\end{abstract}

This paper will attempt to demonstrate that the distribution of college students' memories about their lives as a function of retention interval can be predicted by assuming that the students (1) encoded an equal number of memory episodes each day of their lives and (2) fail to retrieve memories from this pool in the same manner that they fail to retrieve material learned in the laboratory. That is, autobiographical memory and laboratory memory tasks will both be held to follow the same episodic memory retention function.

The quantitative study of autobiographical memory, as well as of many other subjects in psychology, can be traced back to Galton. Here is how he described his method. "My first experiments were these .... I walked leisurely along Pall Mall, a distance of 450 yards, during which time I

I wish to thank John Robinson for providing me with his data, Gregory Lockhead for suggesting the no cue idea, which became Experiment 2, Herb Crovitz, Gregory Lockhead, and John Staddon for their help during various stages of the project. The research was supported by NSF Grant BNS-8101116, as well as by a Biomedical Research and a University Research Council Grant through Duke University. Address reprint requests to David C. Rubin, Department of Psychology, Duke University, Durham, N.C. 27706. scrutinised with attention every successive object that caught my eyes, and I allowed my attention to rest on it until one or two thoughts had arisen through direct association with that object; then I took very brief mental note of them, and passed on to the next object. . . . It was impossible for me to recall in other than the vaguest way the numerous ideas that had passed through my mind; but of this, at least, I was sure, that samples of my whole life had passed before me, that many bygone incidents, which I never suspected to have formed part of my stock of thoughts, had been glanced at as objects too familiar to awaken the attention ... I I accordingly cast about for means of laying hold of these fleeting thoughts, and, submitting them to statistical analysis ... I selected a list of suitable words and wrote them on different small sheets of paper" (Galton, 1879, pp. 151, 152; reprinted in Crovitz, 1970; pp. 25, 26). Galton then observed these words one at a time, recording the associations evoked and his reaction time. Later he noted the period of his life from which the associations had come using three broad time periods. Boyhood and youth accounted for $39 \%$ of the 124 nonrepeating associations, subsequent man- 
hood for $46 \%$, and quite recent events for $15 \%$.

In 1974, Crovitz and Schiffman modified Galton's method slightly: they used more subjects, they chose their stimulus words to be common, picturable nouns, they failed to take reaction times, and they asked for personal memories, not just associations, eventually including in their instructions a version of Tulving's (1972) definition of episodic memory as a recollection that could be described as "I did such and such, at such and such a time, in such and such a place" (e.g., Crovitz \& Harvey, 1979). Their major finding was quite remarkable. Although their procedure might be expected to produce a rather haphazard distribution of responses, they found that the number of memories from any hour of one's life could be expressed as a power function of hours passed since that hour.

Several studies of autobiographical memory have followed the same basic procedure adapted by Crovitz and Schiffman from Galton. Variations in the procedure involve the age of the subjects used (Fitzgerald, 1978, 1980; Franklin \& Holding, 1977; McCormack, 1979), properties of the cue words (Chew, 1979; Fitzgerald, 1978, 1980; Robinson, 1976; Rubin, 1980), the period of the life span from which subjects are instructed to get memories (Chew, 1979; Crovitz \& Harvey, 1979; Crovitz, Harvey \& McKee, 1980; Crovitz \& QuinaHolland, 1976), the imagery ability and other traits of the subjects (Franklin \& Holding, 1977; Karis, 1979), and the questions asked in addition to the age of the episode (Chew, 1979; Fitzgerald, 1978, 1980). The Crovitz-Schiffman function has been confirmed by all but one (McCormack, 1979) of the researchers who considered it (Crovitz \& Harvey, 1979; Crovitz, Harvey, \& McKee, 1980; Crovitz \& Quina-Holland, 1976; Franklin \& Holding, 1977; Robinson, 1976 as reanalyzed here, Rubin, 1980), though it may not hold for some clinical populations (Albert, Butters, \& Levin, 1979).

In spite of the regularity found, many re- searchers who use the Crovitz-Schiffman method to collect data fail to test for, or make interpretations based on, the power function. This may be due in part to the difficulty in following Crovitz and Schiffman's description of their analysis, and to the large number of data points needed for their analysis, but mostly it is due to using the cuing method as a tool to approach other problems. Given the rarity of such quantitative findings in areas of psychology as naturalistic as autobiographical memory, the Crovitz-Schiffman plot is extremely intriguing. It has the potential of affording tests of quantitative models of memory in a more "ecologically valid" domain than is usual. Ignoring a two-parameter function that can explain better than $90 \%$ of the variance in what one would assume is a complex distribution is a luxury that psychology cannot currently afford.

By assuming equal encoding of autobiographical memory over time, the CrovitzSchiffman function can be viewed as a retention function with the decrease in memory over time seen as a failure to retrieve. If the Crovitz-Schiffman function is really a retention curve for episodic memory, how does it compare to retention curves obtained in the laboratory?

The first thing to note about retention is that it seems to be mainly a function of time, so trying to specify a retention curve only as a function of time may be a reasonable first approximation. Perhaps the strongest claim comes from Underwood.

Available data indicate that when the naive $S$ learns a list so that he can recite it just perfectly, his forgetting will be influenced only by the length of the retention interval. No other manipulable variable appears to have any appreciable influence on the forgetting in this situation. ... Won't lists of high-meaningful items be remembered better than a list of low-meaningful items? Won't a list of common words be retained better than a list of difficult trigrams? Apparently not. If both tasks are taken to the same level of learning, forgetting is about the same (Underwood, 1966, p. 566).

Of course, varying the contents of the retention interval is not allowed if this claim 
is to hold, but for autobiographical memory the level of interference should be fairly constant throughout one's life. More recently, Nelson and Vining (1978) have extended Underwood's claim by showing that retention is not affected by varying the orienting task from semantic to structural as long as the level of initial learning is not changed.

If retention is a function of time, what function? Plots of many early experiments showed the retention function to be negatively accelerated. Ebbinghaus straightened the curve out somewhat by taking the log of the time axis (i.e., memory strength $=-\alpha$ $\log t+\beta$, where $t$ is time and $\alpha$ and $\beta$ are positive-valued parameters), a transformation which is still common with timing data. Others, assuming the exponential decay (i.e., $\alpha e^{-\beta t}$ ) common in physical systems where the rate of loss is proportional to how much is left, tried straightening the curve out by taking the $\log$ of the memory strength axis. Crovitz and Schiffman found that taking the log of both the memory strength and the time axis worked best for them, yielding a power function (i.e., $\alpha t^{-\beta}$ ).

The most systematic approach to this problem comes from Wickelgren. Initially Wickelgren (1973) postulated a separate retention function for short and long term memory and found data to support the notion that short term memory could best be described by an exponential decay, while long term memory could be described by a power function decay or an exponentialpower function [i.e., $\alpha e^{\beta t(1-\gamma)}$ ] (Wickelgren, 1972). Although both the power function and the exponential-power function fit the data equally well, the exponentialpower function had fewer unwanted changes in parameters as the range of retention intervals of these experiments varied. Wickelgren $(1974,1975 b)$ was finally able to account for what is normally termed long- and short-term memory with one function, doing away with what he considered a distinction with no empirical base.

Wickelgren's single-trace retention function is an exponential multiplied by a power function (i.e., $\alpha e^{-\beta t} t^{-\gamma}$ ). The exponential factor takes interference into account while the power function factor is purely decay. While the exponential factor is most important at short retention intervals where there is great similarity among the items to be recalled, it also has the effect of lowering the curve at long time intervals, even when there is little similarity among the items. It is this slight downward bend at long time intervals that could play a role in autobiographical memory. The exponential factor also makes the switch from the simpler two-parameter power function to the more complex three-parameter exponentialpower function unnecessary for the longer time intervals.

Three observations on the single-trace function are in order. First, it has three parameters as opposed to two for the linear, logarithmic, exponential, or power function, allowing it more freedom in fitting curves. Second, in the form presented, both the single-trace and the power function have values of infinity at $t=0$. This is easily remedied by replacing $t$ by $1+\eta t$ giving the function a value of $\alpha$ at $t=0$. Unfortunately, an additional constant, $\eta$, is introduced. As times close to zero will not be included here, the simpler form given will be retained. Third, although Wickelgren fails to mention the observation, the single-trace function is a gamma function, and thus one that has been extensively studied and might be derived in ways other than Wickelgren's initial formulation.

Examining the data Wickelgren fit to the single-trace function and the data Crovitz and Schiffman fit to the power function it is clear that all the data presented would provide a quite good fit to either curve. The observation that for long delays the points on Crovitz and Schiffman's plot fall below a power function indicate that Wickelgren's single-trace function might be helpful. Crovitz and Schiffman tried curves until one fit the data. The first experiment will repeat Crovitz and Schiffman's procedures in order to provide a more detailed independent test of their results than has been 
previously performed, and in order to compare their empirical function with the more theoretically motivated one that Wickelgren has proposed.

\section{EXPERIMENT 1}

\section{Method}

Subjects. Of the 48 Duke undergraduates who took part, 27 were 18 years old and all ranged from 17 to 21 .

Procedure. The following typed instructions constituted the first page of the booklet used. "This is a study of autobiographical memory, that is, of memory for events in your life that you can specify as occurring at one particular place and time. Shortly, I will ask you to inspect each of the words on the following pages, one at a time, until a specific memory associated with that word comes to mind. As soon as you think of such a memory, you should write it down in the space provided. One or two words should be sufficient, so that you can later refer back to what you wrote. If a specific memory does not come to mind for a word within 10 to 15 seconds, please put an ' $\mathrm{X}$ ' beside that word and go on to the next word." The next few pages contained 125 words displayed one word per line. Twenty-four random orders of these words were generated and printed by a computer, one for each of 24 subjects. The remaining 24 subjects each had the reverse of these orders. Following completion of the first part of the experiment, the subjects were asked to break a tape seal and read the following instructions. "For each of the memories that you have listed, tell how long ago the event occurred, to the best of your recollection (for example: $10 \mathrm{sec} ., 30$ min., 1 hr., 3 days, 5 months, 7 years). Please indicate your times to the left of each word. Please give a single time, not a range of times."

\section{Results}

An analysis of the data in terms of the average age and number of memories provided for each of the 125 words is available in Rubin $(1980,1981)$. A total of 4855 dated memories were obtained from a possible maximum of 6000 . The missing data are primarily due to certain stimulus words, such as "context," "glacier," and "virtue," not evoking many memories in the college students.

The following analysis was used to transform the 4855 dated memories into a distribution of memories per hour as a function of hours ago. Many of the decisions made by Crovitz and Schiffman (1974) and followed here are arbitrary. A different response measure, developed by Robinson (1976), which avoids many of the problems encountered with the method to be presented here, will be used in the next experiment. The basic ploy Crovitz and Schiffman used to convert the set of discrete points to a distribution was to assume that when someone used an English time marker such as minute, day or year, they implied an accuracy of $\pm 1 / 2$ of that time marker. Thus, 3 years ago really means $2 \frac{1}{2}$. to $3 \frac{1}{2}$ years ago while, 35 months ago really means $341 / 2$ months ago to $351 / 2$ months ago. In this way, memories are divided across a span equal to the width of the time marker.

This system seems like a reasonable approximation, except that certain round numbers, which seem to imply less accuracy, have frequency higher than that of surrounding responses. Thus, in the data collected here and in Crovitz and Schiffman's data, 10 years ago, which seems to imply less accuracy than 9 or 11 years ago, has a greater frequency than 9 or 11 years ago. Schiffman (personal communication) has suggested that this flaw could be remedied by obtaining independent judgments of the accuracies implied by the various timing terms used.

Given this system, a distribution of memories per hour as a function of hours ago was formed by dividing the frequency of each response (such as 5 days ago) by the number of hours in the time marker (24 hours for $\pm 1 / 2$ day), and incrementing the distribution by this value over the appropri- 


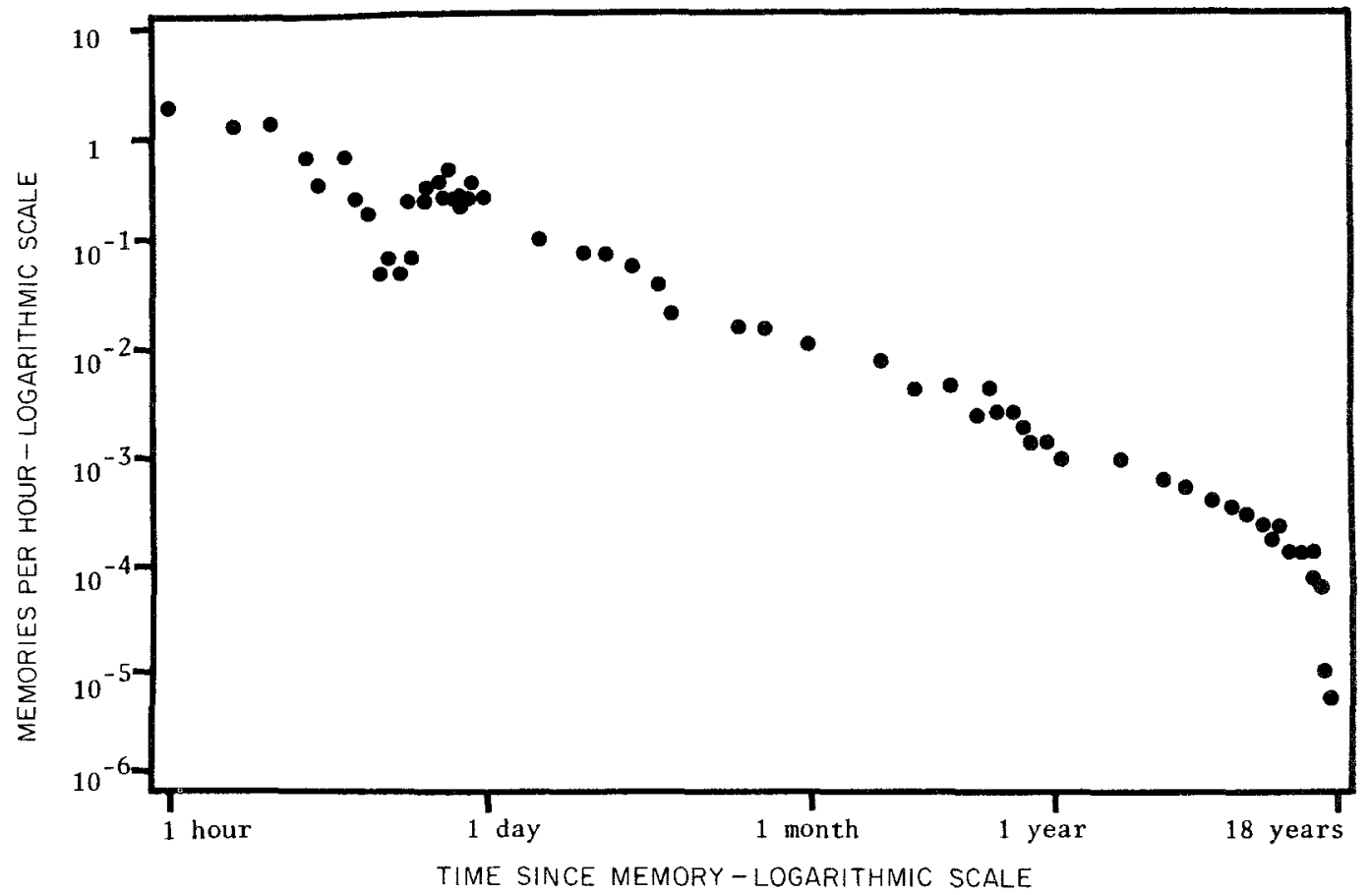

FIG. 1. The mean number of memories per hour reported from the subjects' lives as a function of the age of those memories. The vertical axis assumes a total of 100 reported memories. One point, zero memories per hour at 18 years, is not plotted.

ate range $\left(4 \frac{1}{2}\right.$ to $5 \frac{1}{2}$ days ago). This distribution was sampled at the 61 points noted by the time markers of English which span the range from 1 hour ago to 18 years ago: $1,2, \ldots, 23$ hours, $1,2, \ldots, 6$ days, $1,2,3$, weeks, $1,2, \ldots, 11$ months, and $1,2, \ldots$, 18 years. Points less than an hour ago were not included because people tended not to list episodes that recent. This is probably because such episodes do not seem to qualify as "events." For example, in this experiment only 61 of the 4885 memories were less than 1 hour ago. Nonetheless, many of these 61 responses actually did enter into the calculations because the range for the responses $1 / 2$ and $3 / 4$ hour ago included 1 hour ago. Following this method: responses of 1 day ago would contribute to the value for $13,14, \ldots, 23$ hours ago as well as 1 day ago; responses of $1 \frac{1}{2}$ days ago would contribute to both 1 and 2 days ago; and responses of 39 days ago would contribute to none of the 61 points sampled, because its range ( $381 / 2$ to $391 / 2$ days ago) did not include any of the 61 points used.

The experiments performed here will have different numbers of total dated memories. In order to normalize the plots to make them more comparable, each of the memory per hour values reported in this paper has been divided by the total number of dated memories in its experiment, and then multiplied by 100 , as if there were 100 memories in every experiment.

Figure 1 presents the values obtained plotted on $\log -\log$ paper.

One of the 61 points, zero memories per hour at 18 years ago, was not plotted or included in any of the calculations, because the $\log$ of zero is minus infinity. The two adjacent points, those at 16 and 17 years ago, are well below the curve as plotted in Figure 1, possibly indicating a lack of memories for the first 3 years of life which is distinct from normal forgetting. While the claim of childhood amnesia for the first 3 
years of life would require defending the power function used here over other functions which could provide a continuous plot through all 61 points (Wetzler, Note 1), it seems most reasonable at present to perform the major analyses with the values of 16 and 17 years ago excluded.

Figure 1 is a plot of memories per hour as a function of hours ago. Both scales are logarithmic, so a straight line would indicate a power function. The best leastsquares power function not including the values for 16,17 , or 18 years ago is $\log$ (memories per hour) $=-.82 \log$ (hours ago) $+.34(r=-.983)$; while the best singletrace fit is $\log$ (memories per hour) $=-.77$ $\log$ (hours ago) $-3.10 \times 10^{-6}$ hours ago + $.27(r=.985)$. Wickelgren usually provides his analyses in terms of seconds, not hours. For seconds ago, the last two constants would change to $-8.60 \times 10^{-10}$ and 3.02 . If the 16- and 17-year points are included, the correlations for the power and the singletrace functions drop to -.974 and .982 , respectively. Thus, both functions fit the data extremely well, with little to differentiate between them empirically. The parameters estimated are in good agreement with past experiments. The power function exponent of -.82 is extremely close to Crovitz and Schiffman's obtained value of -.78 .

Several commonly proposed two-parameter retention functions will not fit the data nearly as well as the power or the singletrace function. Again, with the 16-, 17-, and 18-year-ago points excluded the linear, logarithmic, and exponential decays provide correlations of $-.294,-.635$, and -.767 , respectively. These correlations are not less than .98 because of noise, but because of systematic curvilinear trends which are not present with the power or single-trace function. On the other hand, other twoparameter functions including Wickelgren's exponential-power function with $1-\gamma=$ .25 and the hyperbolic function (i.e., $\alpha / 1+$ $\beta t$ fit nearly as well with correlations of -.955 and .946, respectively, as does a parabola of the form $\log$ (memory per hour) $=\alpha(\log t)^{2}+$ $\beta \log t+\gamma(r=.984)$. Decisions among these functions are more difficult to make on empirical grounds with the data presented here, but could rely on other properties of the curves and their relation to theories of retention.

\section{EXPERIMENT 2}

The results of Experiment 1 are encouraging, but the method has some flaws. First, a complex set of somewhat arbitrary rules was needed to transform the data into a memory distribution. Second, the cue words used are known to influence the dating of the memories evoked (Chew, 1979; Robinson, 1976; Rubin, 1980) and therefore, could have affected the shape of the retention function. Third, the subjects varied considerably in age, making interpretations of the 16-, 17-, and 18-yearago points more difficult. Experiment 2 will attempt to overcome these flaws and provide another evaluation of the retention functions proposed.

Robinson (1976) had subjects indicate the age of their memories by indicating the year, months, and any other information that they could supply concerning the actual date and time. This dating proved reliable. The correlation between the first dating of the evoked episodes and a second dating one week later was .94. While this method takes longer for the subjects to use, it avoids the problem of having to make a continuous distribution out of the data that bunches up at 60 or so standard time markers.

Cue words were first used by Galton and followed by everyone since. They may, however, not be a necessity. People should be able to list episodes from their lives without external cues, just as they can list semantic domains (Rubin \& Olson, 1980). This could lead to increased clustering in the Bousfield sense, but this need not be undesirable as long as the number of clusters produced is large enough to provide a representative sample of memories.

\section{Method}

Subjects. The 84 undergraduates who 
took part were all 18-year-olds at the time of the experiment.

Procedure. A booklet contained the following instructions:

"On the following 2 pages please record 50 events from you life that could be written in the form 'such and such happened at such and such place at such and such time.' The events need not be noteworthy. Just let your mind wander until you happen on them. Please record one event beside each number. All you need write down is a few words to remind you later in the session of the particular event you thought of. These words need not be intelligible to anyone else besides you. The only identifying information that will be asked for is your age and sex. Please excuse the openendedness of the question, but I do not want to bias your choice in any way."

After recording 50 events, the subjects were instructed to break a tape seal which revealed the following instructions adapted from Lindsay and Norman (1972, p. 379).
"Please go back to each event and date it as accurately as you can in terms of the year, month, day, and hour it occurred using the form that follows this sheet. The dating will take some thinking, but is not as difficult as it first seems. For example, for an event that occurred around ten years ago: What grade of school were you in? What season was it? Was it on or near a holiday or someone's birthday? Was it morning or afternoon? Try to be as accurate as you can be, but if all you can remember is something like: 'It was a spring afternoon when I was seven or eight,' then put down your best guess at the year, month, day, and hour, with a question mark after each number that you just took a guess at. Please note that combinations like being quite sure of the hour without knowing the year are possible.

"To help give you some benchmarks to compare your events with please record the following dates: date of birth, 3rd birthday, entered 1st grade, left 1st grade,... left

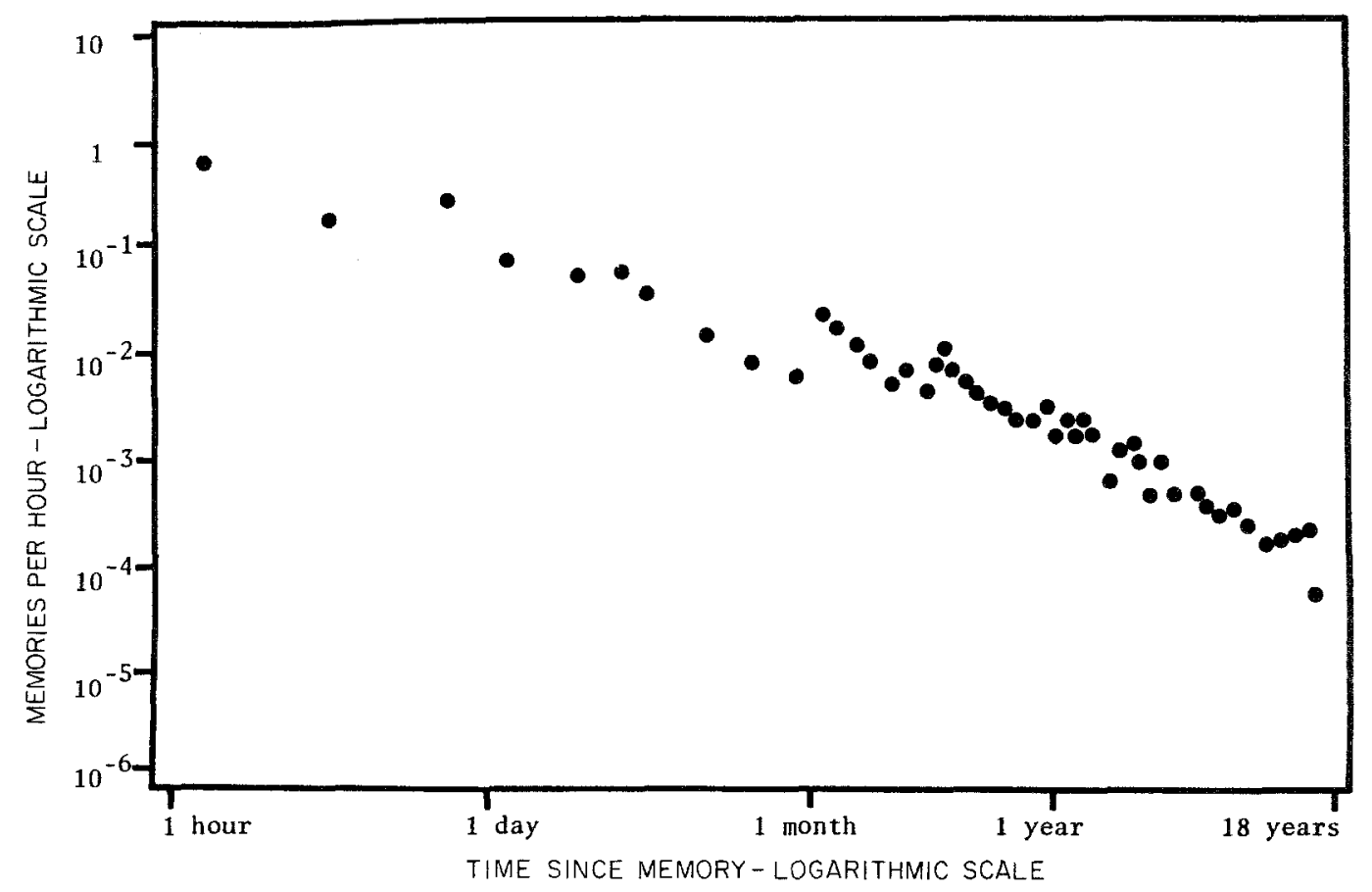

FIG. 2. The mean number of memories per hour reported from the subjects' lives as a function of the age of those memories. The vertical axis assumes a total of 100 reported memories. 
12th grade, and any other events from you, your family or firends that could help your dating such as moves, births, marriages. Again feel free to use codes intelligible only to you." Subjects were provided with the date of the experiment and a sheet containing 50 blank dates with columns for year, month, date, and hour.

\section{Results}

A total of 4169 dated episodes were obtained from a possible maximum of 4200 . In order to collapse these data into points comparable to those of Experiment 1, 48 points of 85 episodes each and 1 point of 89 episodes were formed. The median of each of these 49 groups of episodes was used to determine the middle point of the group, and the range was used to determine the number of memories per hour. As in Experiment 1 , the memories per hour were divided by the total number of memories and multiplied by 100 , as if a total of 100 memories were obtained in the experiment.

Figure 2 presents the 49 values obtained plotted on $\log -\log$ paper.

As with Figure 1, there is some indication of a drop off for the oldest memories. In this case it is the last point (covering a range from birth to 5 years old) which does not appear to be continuous with the rest of the data. The period of the drop-off is greater here than in Experiment 1 because 89 memories had to be accumulated from a period in which there were not many memories. Most of these came from the older end of the interval, the median of these 89 memories being 4 years old. As with Experiment 1 , this last point will not be included in the main analyses.

A straight line on Figure 2 would represent a power function. The best fitting power function is $\log$ (memories per hour) $=-.76 \log$ (hours ago $)+.22(r=-.975)$, while the best single-trace fit is $\log$ (memories per hour) $=-.66 \log$ (hours ago) $-6.00 \times 10^{-6}$ hours ago $-.01(r=.984)$. In terms of seconds ago, the last two constants would change to $1.67 \times 10^{-9}$ and 2.36 . If the last point is included, the correlations for the power and single-trace theory functions become -.972 and .985 , respectively. As with Experiment 1, both functions fit the data extremely well, with little to differentiate between them. The parameters estimated are in good agreement with earlier values as well as with Experiment 1. In particular, the slope for the power function is in the range of earlier work, and the exponential parameter of Wickelgren's function is quite small as it should be in this experiment. The other two parameters of Wickelgren's function are harder to compare to his earlier work, because of differences in methods.

Again the linear, logarithmic, and exponential decays do not fit as well as either the power or the single-trace functions, with correlations of $-.197,-.671$, and -.749 , respectively, while the exponential-power hyperbolic and parabolic functions do, with correlations of $-.965, .982$, and .984 , respectively.

\section{DISCUSSION OF EXPERIMENTS 1 AND 2}

The first two experiments used different methods to cue episodes as well as different response measures. In the first experiment each of 125 words was used to cue individual memories, while in the second experiment 50 episodes were listed without any external cues. In the first experiment subjects dated their episodes by recording a number plus time marker (e.g., 2 days ago), while in the second their best estimate of year, month, day, and hour were recorded. Although the methods varied, the results were the same, making an explanation in terms of methodological artifacts less likely.

The results of both experiments fit a power function, replicating Crovitz and Schiffman as well as much of Wickelgren's work with memories longer than a minute. Wickelgren's single-trace function provides a somewhat better fit, but logically it must be so. This is because Wickelgren's function has an extra parameter and in fitting 
the data this parameter enters in with a second independent variable (hours ago) in a multiple regression equation. At worst it could add nothing. If we were to assume that the 58 points used in Experiment 1 and the 48 used in Experiment 2 were independent, which they are not, then the parameter of these exponential factor added by Wickelgren to the power function would be significantly different from zero in the two experiments, $F(1,55)=5.54$, and $F(1,45)=$ 27.15 , respectively. Of more importance, the exponential factor acts in a systematic way. If the data of Experiment 1 and 2 are fit with a power function, the oldest 5 points of Experiment 1 and the oldest 10 points of Experiment 2, (not counting the points excluded from the main analyses) would fall below the regression line. That is, the residuals do not appear to be distributed randomly, but systematically. The addition of the exponential factor makes the residuals appear randomly distributed. The pattern of results is the same as that Wickelgren reports in his studies of retention under more controlled circumstances (Wickelgren, 1974, 1975b). According to Wickelgren, the exponential factor represents interference as opposed to decay. Even though this interference is small in autobiographical memory, the cumulative effect over a lifetime is large enough to produce a noticeable deficit.

If the points from near birth are included in the analyses, Wickelgren's function has more of an empirical advantage. For Experiments 1 and 2 , including these points does not decrease the correlations with Wickelgren's function. They change from .985 and .984 to .982 and .985 , respectively. Thus, Wickelgren's function can fit curves with points that, on $\log -\log$ paper, appear to be below a continuous straight line function. It may be noted that if childhood amnesia is defined as a drop below the normal retention function (Wetzler, Note 1), then for Wickelgren's function, no evidence for childhood amnesia has been collected here. The one zero frequency point at 18 years ago from Experiment 1 not included in the analyses may be seen as some indication that childhood amnesia exists for at least the first year of life, but if more subjects were run, a nonzero value might be obtained for this point and that value might also fit on Wickelgren's curve.

The choice between the power and single-trace function, cannot be purely an empirical one. The power function will account for most of the variance. While adding the exponential factor to produce the single-trace function adds some systematic explanatory power, it also adds an extra parameter. The main advantage of the addition of the exponential factor is that it provides a tie between the autobiographical memory literature and that of more controlled studies of laboratory learning. Two cautions concerning the addition of the exponential factor are in order. First, unless enough data are collected to provide a smooth empirical curve, the role of the exponential factor at long delays is small enough that it will not be noticeable, even in laboratory studies (Wickelgren, 1975a). Second, it is possible that the drop below the power function decay accounted for by the exponential factor may be due to different causes in the studies performed here, and in those performed by Wickelgren. However, given the similarity in the form of the functions in the various studies, it is difficult to pursue this possibility without further empirical manipulations.

The choice between the power and single-trace functions is one instance of a more general problem. The retention data from list learning and autobiographical memory are quite similar, and at least two functions fit both sets of data quite well. However, there exist many functions that might fit the data if they were tried. That is, given one or two free parameters, many functions can mimic one another over limited ranges. Even logarithmic and linear functions can be difficult to separate empirically (Rubin, 1976).

Of the vast number of commonly used 
mathematical functions that could provide the negatively accelerated, monotonically decreasing plot necessary for a retention function, psychologists tend to concentrate on the exponential family used for physical decay processes. The hyperbolic function gets little attention in any kind of recall process (see Bousfield, Sedgewick, \& Cohen, 1954; or Gruenewald \& Lockhead, 1980, for exceptions). Here it provides an excellent fit for the first two experiments. Unlike the single-trace function and power function, however, it provides a much poorer fit if the long retention interval points dropped from the main analyses are added back in. The .946 and .982 correlations from Experiment 1 and 2 drop to .546 and .877 , respectively, when these points are included.

The parabolic curve $[\log$ (memories per hour) $\left.=\alpha(\log t)^{2}+\beta \log t+\gamma\right]$ also provides an excellent fit, and one which declines only slightly if the long retention points are included. The .984 and .984 correlations from Experiment 1 and 2 drop only slightly to .978 and .983 , respectively. The parabola's success is not due simply to the $\log t$ component, as the beta weight on the $(\log t)^{2}$ and the $\log t$ components are roughly equal to each other in the two experiments. An exceedingly large number of functions as seemingly reasonable as a parabola in $\log t$ could probably be fit to the data with equal success. There are reasons for considering the parabola, however. If the distribution of memories per hour is lognormal, then the logarithm of the memories per hour values would be described by the parabola given earlier in this paragraph (I owe this observation to Davis Howes). As the lognormal distribution has only two free parameters, $\mu$ and $\sigma$, the value of the intercept, $\gamma$, can be calculated in terms of $\alpha$ and $\beta$. Unfortunately, when $\alpha$, $\beta$, and $\gamma$ are all free to vary, the predictions of $\gamma$ from $\alpha$ and $\beta$ are not good. Nonetheless, the parabolic function serves to demonstrate that there may be many theoretically motivated functions besides Wickelgren's that could be formulated to account for the data presented.
So far, this paper has considered the decisions among retention functions as a problem in curve fitting. Initially, this is useful because many functions can be eliminated this way, including some commonly assumed to be of value. Choosing among functions that are similar over the range of times considered here, however, is no longer a curve-fitting question, but rather one that requires other methodological and theoretical decisions.

The contribution of the first two experiments, when taken with Wickelgren's work, is to provide some empirical constraints on what kinds of functions will work. Other constraints can also be collected. Jost's second law (Simon, 1966; Wickelgren, 1972) is a favorite. Boundary conditions, such as a finite number of memories per hour at $t=0$ and zero memories per hour at $t>$ the lifetime of the subject could also be added. By comparing these and other yet to be discovered empirical observations with the properties of the functions, a decision among functions could be made. It would be more desirable, however, to formulate a more general theory of memory which would postulate an adequate retention function. The uniqueness of the retention function would then no longer be an important question. Instead, the theory that generates the function would be tested using relevant empirical manipulations. One goal of this paper is to help provide an empirical base for such a general theory. In particular, the retention function generated by the theory should not be linear, logarithmic, or exponential. It should be empirically similar to Wickelgren's single-trace function, which fits both autobiographical and laboratory retention. Possible functions beside the single-trace function are quite diverse mathematically, including power, exponential-power, hyperbolic, and parabolic functions of the form presented here.

\section{a Consideration of Possible ARTIFACTS}

The data presented so far were averaged over cues (or a lack of them) and individu- 
als. As either form of averaging could have produced the function obtained, both forms of averaging should be examined. In addition, none of the episodes recorded has been verified and none of the dates checked. The remaining experiments will try to deal with these problems.

In trying to avoid averaging over cues or subjects, it will be necessary to make plots based on less data. Data collected by Robinson (1976) provide an ideal example to demonstrate a histogram technique developed for this purpose, while allowing data from a different laboratory to be examined. Robinson had 24 undergraduates provide descriptions of episodes to 16 object words, 16 activity words, and 16 affect words. The resulting episodes were dated to at least the nearest year and month, and provided 369 , 363 , and 373 dated memories for the three cue word categories. Robinson was kind enough to supply his dates to me in terms of months ago.

The data from each of Robinson's three categories were collapsed into roughly equal intervals on a logarithmic scale 1,2 to 3,4 to 7,8 to 15,16 to 31,32 to 63,64 to 127 , and 128 to 216 months ago. Only the last interval breaks the geometric progression of a logarithmic scale because it is stopped at 18 years ago-the approximate age of the average subject. Episodes dated within the same calendar month as the experiment were recorded, but because they could have spread over such a wide range of logarithmic time, they were not included in the calculations. The number of memories in each interval was counted, and as in the first two experiments the number of memories per hour calculated. To allow for easy comparison, the number of memory per hour figures were divided by the total number of memories in each group and multiplied by 100 , as if there were 100 memories in each group. This normalization also has the effect of leaving the twoparameter power function with only one free parameter to vary.

Given the limited number of points plotted no attempt will be made to try to distin- guish the power and single trace functions; only the simpler power function will be considered. The best-fitting power functions for the three sets of data are $\log$ (memories per hour) $=-.73 \log$ (hours ago) $+.05(r=-.973), \log$ (memories per hour) $=-.83 \log$ (hours ago $)+.43(r=-.990)$, and $\log$ (memories per hour) $=-1.18 \mathrm{log}$ (hours ago $)+1.77(r=-.978)$, respectively. The steeper slope for the affect cues agrees with Robinson's finding of more recent memories for affect cues. Thus, Robinson's data offer support for the type of function found in the first two experiments.

A similar histogram technique can be used for data collected using the Crovitz and Schiffman dating technique. The lifespan from 1 hour ago to 18 years ago is divided into 10 equal logarithmic divisions. All dated episodes are spread out over an interval of $\pm 1 / 2$ their time markers as was done in Experiment 1, and the proportion of that interval that falls in each of the $\mathbf{1 0}$ divisions is credited to that division. An 11th interval containing memories that occurred less than 1 hour ago can be included for completeness, but cannot be included in calculations because its location on the $\log$ hours ago scale is indeterminate.

Using equal intervals on a logarithmic scale means that the last few intervals will be quite large on a linear scale. The distribution of memories within these large intervals was examined in the Robinson data and in all other experiments. No discrepancies with the results of the first two experiments were noted.

\section{EXPERIMENT 3}

Averaging over distributions of one form can produce distributions of another form (Rubin, 1979). This could be the case where distributions of memories per hour as a function of hours ago are averaged over different cue words. An extension of this argument could be made for self-cuing. The fact that different cue words evoke episodes of different ages (Chew, 1979; Robinson, 1976; \& Rubin, 1980) makes this possibility worth examining. The reanalysis of 
Robinson's data just performed indicates that groups of cue words of the same type do produce retention functions of the form found in the first two experiments, but it says nothing about individual cue words.

\section{Method}

Subjects. Of the 212 subjects tested, 132 were 18 years old and all were between 17 and 21.

Procedure. Five words were chọsen from the 125 words used in Experiment 1. The 125 words from that study were rank ordered by their geometric mean age of memory. From each $1 / 5$ of this rank ordering, the word which produced the least missing data was chosen. These five words, the geometric mean age of the memories they evoked, and the percentage of subjects for which the words evoked a memory are: paper, 10 days, 92\%; plant, 25 days, 96\%; wine, 44 days, 100\%; hospital, 61 days, $98 \%$; and fire, 334 days, $100 \%$. Five random orders of these words were made with each word appearing in each serial position once. Approximately one-fifth of the subjects received each order.

The instructions were similar to those used in Experiment 1 with subjects first recording five episodes and then dating them using Crovitz and Schiffman's method.

\section{Results}

The histogram method described earlier was used to group the data into ten intervals of equal size on a logarithmic scale ranging from 1 hour ago to 18 years ago. For the five words, 152, 162, 172, 190, and 159 dated episodes entered into the histograms.

TABLE 1

Parameters of Power Functions

\begin{tabular}{lccc}
\hline Cue word & $\alpha$ & \multicolumn{1}{c}{$\beta$} & \multicolumn{1}{c}{$r$} \\
\hline Paper & -.78 & .10 & -.972 \\
Plant & -.72 & -.08 & -.971 \\
Wine & -.64 & -.34 & -.955 \\
Hospital & -.65 & -.30 & -.995 \\
Fire & -.57 & -.62 & -.981 \\
\hline
\end{tabular}

One interval was left out of the calculations for the word "fire" because it had no episodes. Table 1 presents the values for $\alpha$ and $\beta$ in the equation $\log$ (memories per hour) $=\alpha \log$ (hours ago) $+\beta$. The standard errors for the $\alpha$ values are between .02 and .07 .

From the parameters and correlations given in Table 1 it is apparent that not only is averaging over cue words not necessary to produce power function, but also that the parameters associated with that power function change in an orderly fashion.

\section{EXPERIMENT 4}

A second possible artifact which could yield the retention function found here is averaging over people. Different people could recall episodes from different periods of their lives which average to the distribution described in the first three experiments. That is, the group function may not be representative of the individuals in the group. To investigate this possible problem, data were collected from individual subjects.

\section{Method}

Subjects. Ten 18-year-olds took part in the experiment.

Procedure. The 125 words used in Experiment 1 plus 201 additional words drawn randomly from Paivio, Yuille, and Madigan (1968) were arranged in a random order and typed one per line on the pages of a booklet. Half the subjects saw this order and half saw the reverse order. The instructions were similar to those of Experiment 1.

\section{Results}

The data for the seven subjects who provided dated episodes for more than twothirds of the 326 cue words were analyzed using the histogram method described earlier. Three of these seven subjects had memories in all 10 intervals, three had no memories in one of the first two intervals ( 1 to 3 and 3 to 11 hours), and one subject had no memories in either of the first two inter- 
TABLE 2

Parameters of Power Functions for Individual Subjects

\begin{tabular}{ccccc}
\hline$\alpha$ & $\beta$ & Dated episodes & Intervals omitted & $r$ \\
\hline-.87 & .48 & 288 & 0 & -.980 \\
-.70 & -.10 & 241 & 0 & -.983 \\
-.70 & -.12 & 245 & 0 & -.957 \\
-.68 & -.17 & 299 & 1 & -.928 \\
-.64 & -.42 & 275 & 1 & -.748 \\
-.50 & -.94 & 288 & 2 & -.943 \\
-.48 & -1.01 & 289 & 1 & -.966 \\
\hline
\end{tabular}

vals. These zero memory per hour intervals were not included in the calculations because the logarithm of zero is undefined and it is difficult to decide on a suitable substitute value. The curves for these subjects appear normal in other ways, possibly indicating that their definition of memory did not include recent events. It is more likely, however, that as the intervals left out are quite short and require a minimum number of episodes to provide a reasonable memory per hour figure, this omission is due simply to sampling problems. In particular, the predicted log (memories per hour) per 100 memories in the first interval is less than the intercept of the power function, $\beta$, given in Table 2. For the four subjects with missing intervals, this amounts to approximately $4,2,1$, and 1 memories in each of the first two intervals with the number of memories collected here.

The values for $\alpha$ and $\beta$ in the equation $\log$ (memories per hour) $=\alpha \log$ (hours ago) + $\beta$, the number of events dated, the number of missing intervals, and the correlation to the power function for the seven individual subjects ordered by their slopes are given in Table 2. The standard errors for the seven slopes are $.06, .05, .08, .10, .21, .07$, and .05 , respectively. Given the high correlations, it is unlikely that the retention function is an artifact of averaging over individuals with functions unlike the group function.

The design of this experiment was not intended to test for individual differences in parameters. Such individual differences, however, do appear large. Using the eight intervals for which all subjects provided data, if the eight points from each subject were assumed to be independent observations then an analysis of covariance (Walker \& Lev, 1953, pp. 390-393) would indicate that the slopes are different, $F(6,42=$ $5.55, p<.001$. This experiment did not investigate whether differences in the retention function were stable characteristics of the subjects, and if they were stable, whether they reflect individual differences in general memory functioning (Karis, 1979) or just individual differences in the retrieval strategies used for autobiographical memory.

\section{EXPERIMENT 5}

The dated episodes analyzed so far may be memories only in the sense that people say that they remember them. No evidence has been put forth that they are recollections of actual events. Similarly, while Robinson (1976) demonstrated that the datings are reliable, no evidence has been presented that they are valid. In some sense the question of veridicality is not important. Memory usually does not provide us with an accurate description of the past, even where there is no pressure to reconstruct, as there is with autobiographical memory (Greenwald, 1980). The autobiographical memories recorded in the first four experiments are part of the conscious verbal records of peoples' lives on which much of behavior is based. They are important for normal functioning in the world, for setting expectations, for self-evaluation, selfconcept, and so forth. As such, these 
memories are important independent of the external events that they are supposed to be storing. Nonetheless, if the retention function of laboratory studies is to be shown to describe autobiographical memory, it should at least be demonstrated that the dating of the events, and thus the length of the retention interval, is not biased in a way that would affect the results.

In an attempt to investigate this problem, autobiographical memories were obtained from diary keepers. While diaries, themselves, are records of memories, the retention interval between an event and its recording is usually less than a day, making at least the dating accurate.

\section{Method}

Subjects. A questionnaire was administered to all students taking introductory psychology during one semester at Duke. It asked if the students kept a diary and over what period. The questionnaire then requested that the students provide their name and phone number if they would take part in a memory study in which no other person would see their diaries. Remuneration in terms of an unspecified amount of credit toward the experimental subject requirement and/or money was offered.

Of the 394 undergraduate subjects initially questioned, 181 did not keep diaries, and 106 of those that did indicated that they did not wish to take part in the study. Most autobiographical memories in the experiments reported here come from the recent past, approximately half from the last year. Therefore, subjects who would have taken part but who were no longer keeping their diaries were excluded. Of the remaining 48 subjects, the 18 who had kept their diaries the longest were recruited for the experiment. From the 14 subjects who completed the experiment, following all instructions, the 9 who found more than $25 \%$ of their memories in their diaries were analyzed. One of these 9 subjects was male. Three of the subjects were 17,3 were 18 , and 3 were
19 years old at the time of the experiment. The 9 subjects had kept their diaries for an average of 6 years with a range of 3 to 10 years.

Procedure. Experiment 2 was replicated for the initial session except that 100 events were recorded and dated. The dates were collected and the subjects were provided with their 100 descriptions and asked to redate them using their diaries at their leisure. The initial datings were removed to prevent an increase in the accuracy of scores caused by a vague description fitting an event on or near the date recorded (Linton, 1975).

\section{Results}

The initial datings were examined to see if the diary keepers sampled their memories in a fashion similar to that of other undergraduates. The 879 dated episodes, out of the 900 possible episodes, were analyzed in the same way as those of Experiment 2, and a 10-point histogram like those of Experiment 3 and 4 was formed. One point on the histogram, the 3-to 11-hour interval, had no recorded memories. Based on the remaining 9 points, the best-fitting power function is $\log$ (memories per hour) $=-.51 \log$ (hours ago $)-.81(r=.939)$. Thus, the distribution of the initial datings of the memories seems similar in form to that of the other experiments. The slope is somewhat shallower, however, probably because some of the subjects tried to record memories that could later be found in their diaries in spite of instructions to the contrary. A distribution of the memories found in diaries was not made because it would depend more on diary keeping behavior than on memory.

The nine subjects found a total of 513 memories in their diaries. These dates were subtracted from the ones originally given by the subjects. The median dating errors of the nine subjects ranged from -30 days to 0 days with a median of 0 days. Grouping all the subjects' data together, the median 
error is 0 days. Thus, the errors do not seem to be biased in any particular time direction. To assess the magnitude of the errors the absolute value of the errors were calculated. The medians of the absolute errors of the nine subjects ranged from 0 to 37 days with a median of 3 days. Grouping all the subjects' data together, $27 \%$ of the dates were on the correct day, $37 \%$ within a day, $59 \%$ within a week, $74 \%$ within a month, $93 \%$ within a year, and $97 \%$ within 2 years. If these absolute errors are expressed percentages of the elapsed time as measured by the diaries, $25 \%$ of the errors are less than $0.0 \%, 50 \%$ are less than $1.6 \%$, and $75 \%$ are less than $15.2 \%$. Thus, the errors are quite small, and at least the dating of diary keepers is quite accurate.

The largest error found was 3696 days. A description "seeing Niagra Falls," that was originally dated as occurring in 1970 fit a diary entry from 1980 . It is likely that this and some of the other errors were the result of descriptions being less specific than the subjects had originally thought they were when they tried to date them again several days or weeks later (Linton, 1975). This type of error is not likely to be present in the other experiments where the dating follows the recording of the event more closely. One type of error that is likely to occur in the other experiments is apparent here. The distribution of errors as a function of time has a slight peak at one month and a larger peak at one year, indicating that the subjects do use a calendar organization as well as a time continuum to perform their dating (Robinson, 1976). The subject whose data resulted in the largest median errors suffered from this problem with 14 of her 49 possible errors off by approximately 1 year: 13 in the negative direction.

While one can claim that the diary keepers selected for this experiment are different from most other people, the experiment does offer support for the claim that the dating of memories studied in the previous experiments is relatively accurate.

\section{General Discussion}

The five experiments performed here indicate that the distribution of memories obtained from autobiographical memory is quite stable over a wide variety of conditions, ruling out several explanations of the distribution based on possible artifacts. Moreover, the distribution obtained is easily interpreted in terms of laboratory studies of retention. A set of functions that will work as empirical descriptions of retention and a set of functions that will not has been described. The best function to use cannot be determined by the curvefitting approach used here, but must depend on other theoretical and empirical considerations.

Two issues still need to be considered. The first issue is whether the actual values of the parameters obtained here are interpretable. The second issue concerns the nature of the relationship between the sampling of autobiographical memory examined in this paper and the learning of lists in the laboratory to which it has been compared.

In deciding whether the parameters obtained here are of any real value, the power function will be considered. Examining only the power function when many functions fit the data is actually a reasonable enterprise. If the parameters of the power function vary in an interpretable manner, then the data should be orderly enough that the parameters of the other functions would also be interpretable. In addition, by normalizing the data, so that it appears as if 100 dated events were collected in all experiments, only one free parameter remains in the power function, making comparisons among experiments especially easy. Before excluding the single-trace function from discussion, however, it should be noted that the parameter for the exponential decay portion of the single-trace function, which is the portion that makes the singletrace and power function differ, had reasonable small values given the amount of 
interference that might be expected among the autobiographical memories recalled. In addition, the values of the parameters for the exponential decay were similar in the two experiments that provided enough data to allow a comparison $\left(-3.10 \times 10^{-6}\right.$ and $-6.00 \times 10^{-6}$, respectively).

The slope of the power function obtained by Crovitz and Schiffman was - -78 . The slope from Experiment 1, which was modeled after Crovitz and Schiffman's experiment, was -.82 . Crovitz and Schiffman's experiment produced a less steep slope indicating more older memories. This might be expected given the difference in cue words used. Crovitz and Schiffman used 20 high-frequency, high-imagery cue words while Experiment 1 used a broader sample of cue words, and high-imagery words are more likely to produce older memories (Rubin, 1980). The finding that different words systematically evoke memories with different event ages (Rubin, 1980) was supported by the results of Experiment 3 and accounts for the orderly progression of slopes obtained there. The five words had geometric mean ages of memories of 10,25 , 44, 61, and 334 days ago in Rubin (1980), and had slopes of $-.78,-.72,-.64,-.65$ and -.57 in Experiment 3 . Similarly, the reanalysis of Robinson's data provided an orderly change in slope, reflecting the change in the median age in memory across his three categories of cue words. The slopes there being $-.73,-.83$, and -1.18 .

Experiment 2, which used no cue words, produced a slope of -.76 . Perhaps this can be taken as the least biased measure of the slope parameter because no external cues were given. The value of the slopes from Experiment 2 and the other two experiments, which sampled a large number of autobiographical memories (Experiment 1 and Crovitz and Schiffman), are in good agreement: $-.76,-.82$ and, -.78 , respectively.

While the slopes produced by groups of undergraduates appear to be quite stable, individual subjects vary considerably. This individual variation in the slope, but not in the shape of the function, needs to be further investigated. Karis' (1979) finding that subjects with good imagery ability can retrieve older memories more easily than subjects with poor imagery ability indicates that the variation in slope among subjects may be more than a variation in temporary search strategy. On the other hand, people can find memories from specific parts of their lives (Chew, 1979; Crovitz \& Harvey, 1979; Crovitz et al., 1980; Crovitz \& Quina-Holland, 1976; Karis, 1979) and this may account for both the individual differences and the lower, -.51 , slope in the diary study. If the diary keepers tried to find memories that would be in their diaries, they would produce older memories, and shallower slopes.

Thus, the slope parameters seem to vary in an orderly fashion when groups of subjects are employed, allowing quantitative predictions to be made about new groups of subjects. With additional study, this claim might be extended to the slopes obtained from individuals. The individual variability in slopes does provide one technical difficulty. While the product of power functions with different slopes is a power function, the sum of power functions with different slopes need not be. Nonetheless, the distributions of individual subjects in the experiments performed here do sum to approximately a power function.

The second issue to be considered is the relationship between the sampling of autobiographical memory and the learning of lists in the laboratory to which it has been compared. Differences which weaken the analogy between these two tasks could weaken the claim that they share the same retention function. Some apparent differences stem from differences in the number of possible items that could be remembered. In a typical laboratory situation subjects remember about half of the items. In fact, if the overall percentage of items recalled gets too small, say, below $10 \%$, the experiment becomes inefficient and subject to floor effects. In the autobiographical memory experiments performed here, sub- 
jects were asked to report between 5 and 326 memories from a large set: hundreds or thousands of memories by Galton's (1879) conjecture. Thus, after a single session of these two tasks there would typically be a large difference in the number of items which were not initially remembered. Moreover, there would probably be a large difference in the number of items which were not initially remembered, but could be if more items were requested. This makes the autobiographical memory task seem more like a sampling than a memory problem, but the differences may be mostly in amount, not kind. For both tasks, new items would probably be added, given more time, and for both tasks appropriate cuing might provide new items that would not occur spontaneously. Thus, in neither task could the retention function be considered a forgetting function: in both tasks it must be considered a remembering function.

Nonetheless, at the end of an experimental session subjects would probably claim to have recalled everything they could in a list learning experiment, but not in an autobiographical memory experiment. Moreover, Wickelgren's function comes from forced-choice continuous recognition experiments in which the subjects have to decide whether each item is old or new, a choice which should provide a quite sensitive index of any knowledge subjects have, no matter how limited, making additional recall even less likely. Thus, sampling as well as retrieval may have to be included to understand the relationship between the autobiographical memory task and the list learning task, depending on the theoretical perspective being employed. The simplest initial assumption to make about sampling, and one which would not affect the claims made about a single retention function for the autobiographical memory and list learning tasks, would be that memories are sampled with equal probability from among the accessible memories. The observation that similar functions result no matter whether subjects try to record $5,50,100$, or 326 events supports this assumption.
Another possible difference between this study and laboratory studies is introduced by the length of the retention interval. Any living organism changes over time. When we examine retention intervals that extend over a significant portion of an organism's life the confounding of such changes in the organism with the lengths of the retention intervals becomes more obvious, though in reality this confounding holds even over the shortest time. Rather than being considered a problem, these changes in the organism can be viewed as the causes for declining retrieval, possibly because the organism encoded events differently at different ages, or because the changes destroy memories, or because the organism is different at the time of recall from the way it was at encoding and can no longer retrieve stored information. Thus, a retention function can be viewed as a means of discovering changes in an organism that affect memory. This view seems at least as reasonable as pretending that memory operates in a static organism.

The five experiments of this paper are part of a series of demonstrations that recall from naturally occurring learning situations can be studied in an exact and quantitative manner (Rubin, 1975, 1977; Rubin \& Olson, 1980; Rubin \& Kontis, Note 2). Not only is this important in order to extend and test laboratory findings and generalizations to new situations, but also because the phenomena under study are often of importance in themselves. In this case, a quantitative function was studied which describes the distribution of autobiographical memories-memories which largely determine how we think about ourselves.

\section{REFERENCES}

Albert, M. S., Butters, N., \& Levin, J. Temporal gradients in the retrograde amnesia of patients with alcoholic Korsakoff's disease. Archives of Neurology, 1979, 36, 211-216.

Bousfield, W. A., Sedgewick, C. H. W., \& CoHEN, B. H. Certain temporal characteristics of the recall of verbal associates. American Journal of Psychology, 1954, 67, 111-118.

CHEw, B. R. Probing for remote and recent autobiographical memories. Paper presented at the 87th 
Annual Meeting of the American Psychological Association, New York, 1979.

Crovitz, H. F. Galton's walk: Methods for the analysis of thinking, intelligence, and creativity. New York: Harper \& Row, 1970.

Crovitz, H. F., \& Harvey, M. T. Early childhood amnesia: A quantitative study with implications for the study of retrograde amnesia after brain injury. Cortex, 1979, 15, 331-335.

Crovitz, H. F., Harvey, M. T., \& McKee, D. C. Selecting retrieval cues for early-childhood amnesia: Implications for the study of shrinking retrograde amnesia. Cortex, 1980, 16, 305-310.

Crovitz, H. F., \& Quina-Holland, K. Proportion of episodic memories from early childhood by years of age. Bulletin of the Psychonomic Society, $1976,7,61-62$.

Crovitz, H. F., \& Schiffman, H. Frequency of episodic memories as a function of their age. $B u l$ letin of the Psychonomic Society, 1974, 4, 517-518.

Fitzgerald, J. M. Developmental findings in autobiographical memory: Theoretical implications. Paper presented at the 86th Annual Meeting of the American Psychological Association, Toronto, 1978.

Fitzgerald, J. M. Sampling autobiographical memory reports in adolescents. Developmental $P_{S y-}$ chology, 1980, 16, 675-676.

Franklin, H. C., \& Holding, D. H. Personal memories at different ages. Quarterly Journal of Experimental Psychology, 1977, 29, 527-532.

Galton, F. Psychometric experiments. Brain, 1879 , 2, 148-162.

Greenwald, A. G. The totalitarian ego: Fabrication and revision of personal history. American Psychologist, 1980, 35, 603-618.

Gruenewald, P. J., \& Lockhead, G. R. The free recall of category examples. Journal of Experimental Psychology: Human Learning and Memory, 1980, 6, 225-240.

KARIS, D. Individual differences in autobiographical memory. Paper presented at the 87th Annual Meeting of the American Psychological Association, New York, 1979.

Lindsay, P. H., \& Norman, D. A. Human information processing: An introduction to psychology. New York: Academic Press, 1972.

Linton, M. Memory for real-world events. In D. A. Norman \& D. E. Rumelhart (Eds.), Explorations in cognition. San Francisco: Freeman, 1975.

McCormack, P. D. Autobiographical memory in the aged. Canadian Journal of Psychology, 1979, 33, $118-124$

Nelson, T. O., \& Vining, S. K. Effect of semantic versus structural processing on long-term retention. Journal of Experimental Psychology: Human Learning and Memory, 1978, 4, 198-209.

Paivio, A., Yuille, J. C., \& Madigan, S. A. Concreteness, imagery, and meaningfulness value for
925 nouns. Journal of Experimental Psychology Monograph, 1968, 76(1, Pt. 2).

RoBinson, J. A. Sampling autobiographical memory. Cognitive Psychology, 1976, 8, 578-595.

Rubin, D. C. Within word structure in the tip-ofthe-tongue phenomenon. Journal of Verbal Learning and Verbal Behavior, 1975, 14, 392-397.

RuBin, D. C. Frequency of occurrence as a psychophysical continuum: Weber's fraction, Ekman's fraction, range effects, and the phigamma hypothesis. Perception and Psychophysics, 1976, 20, 327-330.

Rubin, D. C. Very long-term memory for prose and verse. Journal of Verbal Learning and Verbal Behavior, 1977, 16, 611-621.

Rubin, D. C. On measuring fuzziness: A comment on "A fuzzy set approach to modifiers and vagueness in natural language." Journal of Experimental Psychology: General, 1979, 108, 486-489.

RuBIN, D. C. 51 properties of 125 words: A unit analysis of verbal behavior. Journal of Verbal Learning and Verbal Behavior, 1980, 19, 736-755.

Rubin, D. C. Norms for properties of words. JSAS Catalog of Selected Documents in Psychology, 1981, 11, 19, ms. 2213.

Rubin, D. C., \& Olson, M. J. Recall of semantic domains. Memory and Cognition, 1980, 8, 354-366.

Simon, H. A. A note on Jost's Law and exponential forgetting. Psychometrika, 1966, 31, 505-506.

Tulving, E. Episodic and semantic memory, In E. Tulving \& W. Donaldson (Eds.), Organization of memory. New York: Academic Press, 1972.

UNDERWOOD, B. J. Experimental psychology. New York: Appleton-Century-Crofts, 1966. 2nd ed.

WALKER, H. M., \& LEV, J. Statistical inference. New York: Holt, Rinehart \& Winston, 1953.

Wickelgren, W. A. Trace resistance and the decay of long-term memory. Journal of Mathematical Psychology, 1972, 9, 418-455.

WiCkelgren, W. A. The long and short of memory. Psychological Bulletin, 1973, 80, 425-438.

Wickelgren, W. A. Single-trace fragility theory of memory dynamics. Memory and Cognition, 1974, 2, 775-780.

Wickelgren, W. A. Age and storage dynamics in continuous recognition memory. Developmental Psychology, 1975, 11, 165-169. (a)

Wickelgren, W. A. Alcoholic intoxication and memory storage dynamics. Memory and Cognition, 1975, 3, 385-389. (b)

\section{REFERENCE NoTES}

1. Wetzler, S. E. Childhood amnesia and early memories: A review of the literature. Unpublished manuscript, Duke University, 1980.

2. Rubin, D. C., \& Kontis, T. C. A schema for common cents: Memory for coins. Unpublished manuscript, Duke University, 1981.

(Received September 3, 1981) 